Check for updates

Cite this: Chem. Sci., 2020, 11, 742

๑ All publication charges for this article have been paid for by the Royal Society of Chemistry

Received 7th November 2019 Accepted 23rd November 2019

DOI: $10.1039 / \mathrm{c} 9 \mathrm{sc} 05627 \mathrm{k}$

rsc.li/chemical-science

\title{
An umpolung approach to the hydroboration of pyridines: a novel and efficient synthesis of $\mathrm{N}-\mathrm{H}$ 1,4-dihydropyridines $\uparrow$
}

\author{
Huan Yang, Li Zhang, Fei-Yu Zhou and Lei Jiao (iD *
}

\begin{abstract}
The first inverse hydroboration of pyridine with a diboron(4) compound and a proton source has been realized under simple basic and catalyst-free conditions. This process consists of a formal boryl anion addition to pyridine, which produces an $\mathrm{N}$-boryl pyridyl anion complex, and the subsequent protonation of the anion complex. This process enables a simple and efficient method for the synthesis of multisubstituted $\mathrm{N}-\mathrm{H}$ 1,4-dihydropyridine (1,4-DHP) derivatives that are difficult to prepare using established methods. Furthermore, this method allows for facile preparation of 4-deuterated 1,4-DHPs from an easily accessible deuterium ion source. This inverse hydroboration reaction represents a new mode for pyridine functionalization.
\end{abstract}

\section{Introduction}

Dihydropyridine (DHP) represents an important class of heterocyclic skeletons which prevalently exist in biologically active agents, pharmaceutically important molecules, and synthetically useful organic reductants, such as nicotinamide adenine dinucleotide (NADH) and Hantzsch ester (HEH). ${ }^{1}$ Owing to their potential medicinal properties and broad application as reducing agents, 1,4-DHP derivatives have attracted great interest in terms of their synthesis. ${ }^{2}$ Reduction of pre-activated pyridines using strong inorganic reductants (Scheme 1a) ${ }^{3}$ and the Hantzsch cyclocondensation (Scheme 1b) ${ }^{4}$ are two conventional synthetic methods of 1,4-DHPs. In recent years, an array of catalytic reduction reactions of pyridines employing milder reducing reagents such as boranes and silanes have been developed (Scheme 1c). ${ }^{5,6}$ In these reactions, pyridine coordinates to a Lewis acid center under catalytic conditions, and then the hydride addition occurs at the $\mathrm{C} 4$ (or C2) position of the in situ activated pyridine.

These hydroboration and hydrosilylation protocols provided ideal and flexible approaches to DHPs from easily available pyridine derivatives utilizing either metal- or organocatalysis. Despite these achievements, alternative methodologies that could produce $\mathrm{N}$-H DHPs in a highly efficient and regioselective manner are still in high demand. Opposite to the Lewis acid activation-hydride addition mode involved in these protocols,

Center of Basic Molecular Science (CBMS), Department of Chemistry, Tsinghua University, Beijing 100084, China.E-mail: Leijiao@mail.tsinghua.edu.cn

$\dagger$ Electronic supplementary information (ESI) available: Experimental procedures, additional information, and characterization data. CCDC 1935576. For ESI and crystallographic data in CIF or other electronic format see DOI: 10.1039/c9sc05627k we envisioned that if the hydroboration of pyridine could be inverse in its polarity, a novel approach to DHPs might be realized. This could not only bring about a conceptually new hydroboration process, but also expand the scope of pyridine reduction. Herein, we report such an umpolung approach to pyridine hydroboration, in which a formal addition of a boryl anion to pyridine occurred first, and the formed Meisenheimer complex was then protonated to form the 1,4-DHP derivative
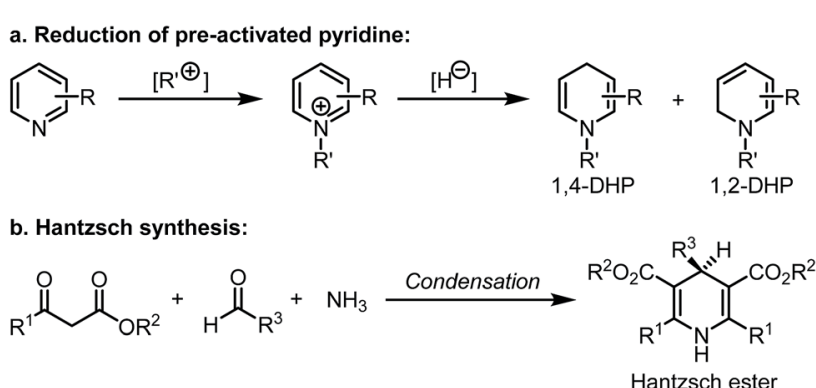

c. Catalytic hydroboration and hydrosilylation of pyridine:

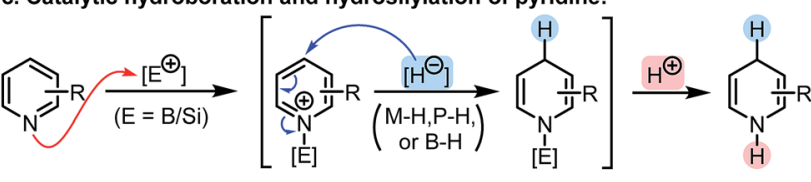

1,4-DHP

d. This work - inverse hydroboration of pyridine:

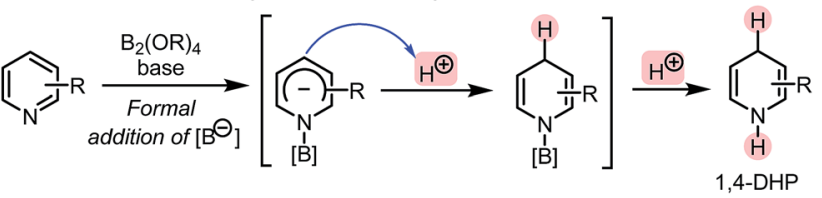

Scheme 1 Methods for the synthesis of 1,4-dihydropyridines (1,4DHPs). 
(Scheme 1d). This anion formation-protonation sequence enables an efficient approach to a variety of $\mathrm{N}-\mathrm{H}$ 1,4-DHPs, which are not easily accessible by conventional methods.

\section{Results and discussion}

\section{Reaction design}

Our idea for realizing the inverse pyridine hydroboration originated from our previous discovery that 4-phenylpyridine (1) efficiently catalyses the borylation of aryl halides with bis(pinacolato)diboron $\left(\mathrm{B}_{2} \mathrm{pin}_{2}\right),{ }^{7 a}$ as well as the related mechanistic studies. ${ }^{7 b}$ It was found that the reaction of pyridine $1, \mathrm{~B}_{2} \mathrm{pin}_{2}$, and a methoxide anion led to the formation of a trans $-2 \mathrm{H}, 2^{\prime} \mathrm{H}-$ [2,2'-bipyridine]-1,1'-diide borate complex 2 (Scheme 2). DFT calculation suggested that the reaction between pyridine 1, diboron, and methoxide first led to the formation of an $\mathrm{N}$-boryl pyridyl Meisenheimer anion (Int-B) through heterolytic cleavage of the B-B bond in the bis-coordinated diboron(4) (Int-A), which was then trapped by another molecule of $\mathbf{1}$ to form complex $\mathbf{2}$. Although Int-B was not observed experimentally, we envisioned to attempt trapping of this proposed anionic intermediate by protonation, which might produce product 3 and thus realize an intriguing umpolung of the conventional pyridine hydroboration process. Further hydrolysis of 3 might afford $\mathrm{N}-\mathrm{H}$ 1,4DHP product 4 (Scheme 2). If realized, such an inverse pyridine hydroboration process would represent a novel mode of pyridine reduction, and bring about new opportunities for the synthesis of 1,4-DHP derivatives.

\section{Proof-of-concept and mechanistic understanding}

We set out to test this hypothesis by employing methanol as a proton source. The reaction between pyridine 1, MeOK, and $\mathrm{B}_{2} \mathrm{pin}_{2}$ was carried out in the presence of 18 -crown- 6 and varied amounts of methanol in tetrahydrofuran (THF). ${ }^{1} \mathrm{H}$ NMR analysis of the crude reaction mixture showed that when 1.1 equivalent of $\mathrm{MeOH}$ was employed, a set of peaks related to dearomatized pyridine emerged (Fig. 1a). Their coupling pattern fitted well with a 4-hydro-4-phenylpyridyl motif, in which the protons in the $\mathrm{R}-\mathrm{CH}(\mathrm{CH}=\mathrm{CH})_{2} \mathrm{~N}$ moiety showed up at $4.25 \mathrm{ppm}$ (broadened triplet, $1 \mathrm{H}$ ), $3.89 \mathrm{ppm}$ (multiplet, $2 \mathrm{H}$ ),

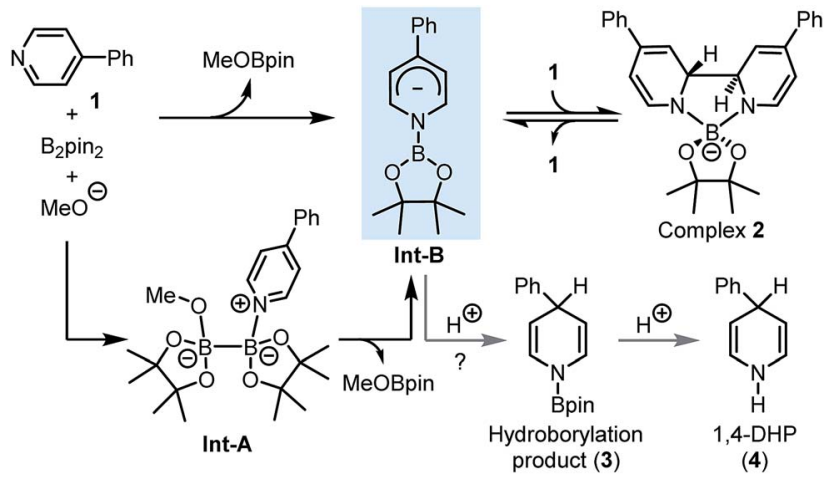

Scheme 2 Design of the inverse hydroboration of pyridine based on our previous mechanistic study.

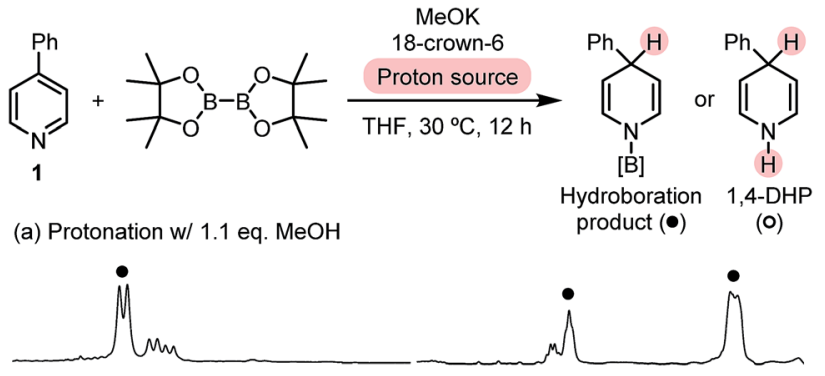

(b) Protonation w/ 1.3 eq. $\mathrm{MeOH}$

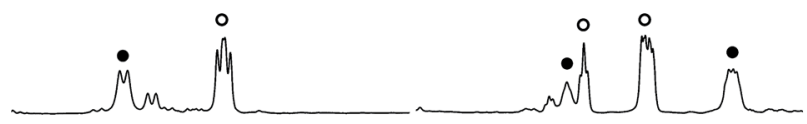

(c) Protonation w/ 2.2 eq. $\mathrm{MeOH}$

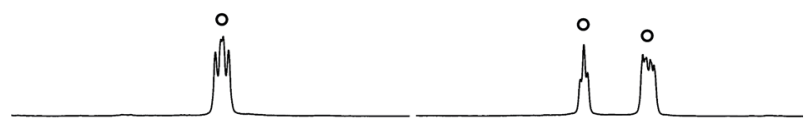

(d) Protonation w/ 2.2 eq. MeOD

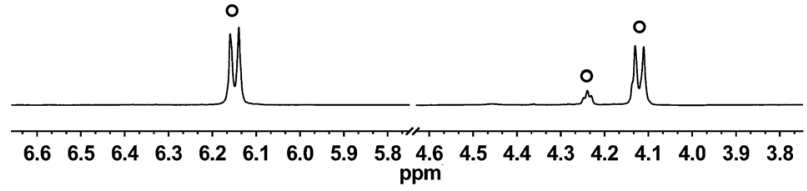

Fig. 1 Partial crude ${ }^{1} \mathrm{H}$ NMR spectra of the $4-\mathrm{PhPy} / \mathrm{B}_{2} \mathrm{pin}_{2} / \mathrm{MeOK}$ reaction system protonated with different amounts of methanol.

and $6.37 \mathrm{ppm}$ (doublet, $2 \mathrm{H}$ ), respectively. Interestingly, with 1.3 equiv. of $\mathrm{MeOH}$, another set of peaks of dearomatized pyridine appeared at $4.21 \mathrm{ppm}$ (broadened triplet, $1 \mathrm{H}$ ), $4.12 \mathrm{ppm}$ (multiplet, $2 \mathrm{H}$ ), and $6.15 \mathrm{ppm}(\mathrm{dd}, 2 \mathrm{H})$, respectively (Fig. 1b), which became the only dearomatized species when excess $\mathrm{MeOH}$ was used (Fig. 1c). The coupling pattern of these peaks was in agreement with that of the desired 1,4-DHP molecule, and gas chromatography-mass spectrometry (GC-MS) analysis of the reaction mixture also detected the peak of the 1,4-DHP product $\left(m / z=157\right.$ for $\left.\mathbf{M}^{\cdot+}\right)$.

When excess deuterated methanol (MeOD) was employed, the methine peak at $4.21 \mathrm{ppm}$ decreased due to deuteration, and the peaks at 4.12 and $6.15 \mathrm{ppm}$ became a doublet because deuterium replaces the coupling protons on both $\mathrm{NH}$ and methane positions (Fig. 1d). This observation, together with the GC-MS analysis of the product $\left[\mathrm{m} / \mathrm{z}=159\right.$ for $\left.\left(\mathrm{M}-d_{2}\right)^{\circ+}\right]$, supported the assignment of the 1,4-DHP structure. These experimental findings served as direct evidence for the formation of the $N$-boryl pyridyl Meisenheimer anion (Int-B) in the diboron(4)/pyridine/base system, and the regioselective protonation at its $\mathrm{C} 4$ position brought about a novel approach to 1,4-DHP derivatives. Because the present reaction is distinct from the established hydroboration protocols in terms of the reaction mechanism, it could be regarded as an inverse hydroboration process.

In order to gain further understanding regarding the formation of the key intermediate Int-B and the regioselective protonation at its C4-position, we performed DFT calculations. In our previous study, the B-B bond cleavage transition state 
(TS1 $\cdot \mathbf{K})$ starting from the bis-coordinated diboron $(4)^{8}$ has been located. ${ }^{7 \boldsymbol{b}}$ In the present study, we analysed the change of NBO charge distribution during the $\mathrm{B}-\mathrm{B}$ bond breaking event (Int$\mathbf{A} \cdot \mathbf{K} \rightarrow \mathbf{T S 1} \cdot \mathbf{K}$, Scheme 3 ). It was found that during this process, negative charge transferred from the MeOBpin part (left part, in blue) to the $N$-borylpyridine part (right part, in red), and the NBO charge on $\mathrm{C} 4$ of the pyridine ring evolved from -0.001 to -0.213 , implying a heterolytic cleavage of the B-B bond with a pair of electrons going to the forming $N$-boryl pyridyl Meisenheimer anion Int-B. The whole process could be viewed as a formal addition of a boryl anion to pyridine, which served as the key step of the inverse pyridine hydroboration. We noted that in the phosphine-catalysed $\beta$-selective hydroboration reaction with $\mathrm{B}_{2} \mathrm{pin}_{2}$ developed by Gulyás and Fernández, a mechanism involving a similar formal boryl anion addition towards conjugated electron-deficient substrates was proposed. ${ }^{9}$ The regioselectivity of the subsequent protonation step was also analysed using the DFT calculated HOMO and the corresponding orbital composition of Int-B (Scheme 3). It was found that $\mathrm{C} 4$ of the pyridine ring had the largest contribution to the HOMO of anion Int-B, which was supported by both the plot of HOMO and the calculated molecular orbital composition using the NAO method. This could account for the observed regioselectivity in the protonation step, which exhibited preference to the formation of 1,4-DHP over 1,2-DHP.

\section{Optimization of reaction conditions and substrate scope}

With this proof-of-concept in hand, we set out to search for suitable reaction conditions for practical synthesis of 1,4-DHP (Table 1). First, diboron(4) compounds with different steric hindrances were attempted, and it was found that the less hindered diboron(4) exhibits better reactivity (entries 1-3), with bis(ethylene glycolato)diboron $\left(\mathrm{B}_{2} \mathrm{eg}_{2}\right)$ leading to the best NMR yield of 4-phenyl-1,4-dihydropyridine (4). A brief screen of bases indicated that $\mathrm{MeONa}, \mathrm{Cs}_{2} \mathrm{CO}_{3}$, and $\mathrm{K}_{2} \mathrm{CO}_{3}$ were all compatible, and among them $\mathrm{Cs}_{2} \mathrm{CO}_{3}$ was the optimal one (entries 4-6).

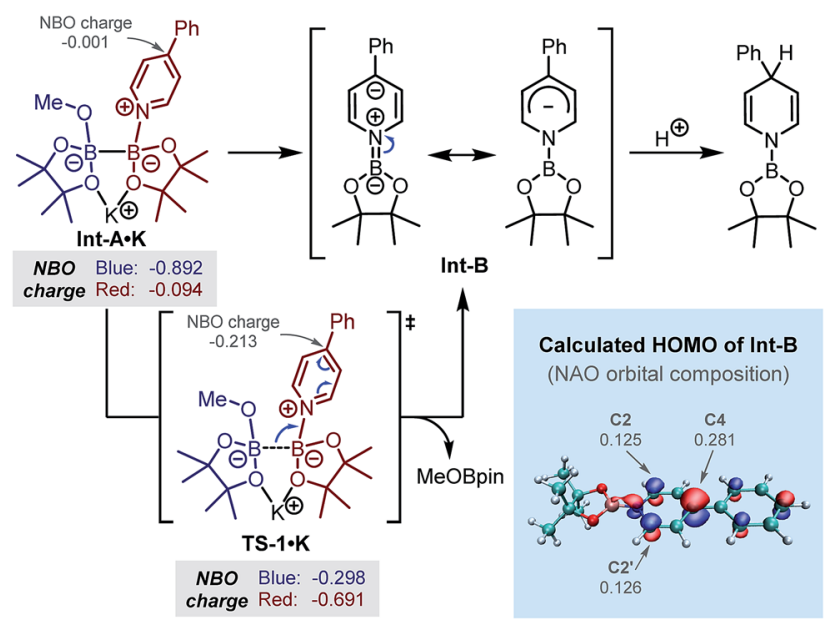

Scheme 3 Mechanism of the formation of Meisenheimer complex Int-B and its highest occupied molecular orbital (HOMO) from DFT calculation.
Table 1 Optimization of reaction conditions ${ }^{a}$

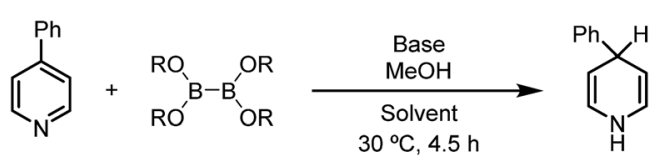

\begin{tabular}{lllll}
\hline Entry & Diboron(4) & Base & Solvent & Conversion $^{b}$ \\
\hline 1 & $\mathrm{~B}_{2} \mathrm{pin}_{2}$ & $\mathrm{MeOK}$ & $\mathrm{THF}$ & $17 \%$ \\
2 & $\mathrm{~B}_{2} \mathrm{neo}_{2}$ & $\mathrm{MeOK}$ & $\mathrm{THF}$ & $63 \%$ \\
3 & $\mathrm{~B}_{2} \mathrm{eg}_{2}$ & $\mathrm{MeOK}$ & $\mathrm{THF}$ & $96 \%$ \\
4 & $\mathrm{~B}_{2} \mathrm{eg}_{2}$ & $\mathrm{MeONa}$ & $\mathrm{THF}$ & $78 \%$ \\
5 & $\mathrm{~B}_{2} \mathrm{eg}_{2}$ & $\mathrm{Cs}_{2} \mathrm{CO}_{3}$ & $\mathrm{THF}$ & $91 \%$ \\
6 & $\mathrm{~B}_{2} \mathrm{eg}_{2}$ & $\mathrm{Cs}_{2} \mathrm{CO}_{3}$ & $\mathrm{THF}$ & $73 \%$ \\
7 & $\mathrm{~B}_{2} \mathrm{eg}_{2}$ & $\mathrm{Cs}_{2} \mathrm{CO}_{3}$ & $\mathrm{MeCN}$ & $71 \%$
\end{tabular}

${ }^{a}$ Reaction conditions: 4-PhPy (0.36 mmol, 1 equiv.), diboron(4) (1.1 equiv.), base (1.1 equiv.), MeOH (11 equiv.), solvent $(2 \mathrm{~mL})$, sealed tube, $30{ }^{\circ} \mathrm{C}$ for $4.5 \mathrm{~h}$. ${ }^{b}$ Determined by ${ }^{1} \mathrm{H}$ NMR analysis. $\mathrm{B}_{2} \mathrm{pin}_{2}=$ bis(pinacolato)diboron; $\mathrm{B}_{2} \mathrm{neo}_{2}=$ bis(neopentyl glycolato)diboron; $\mathrm{B}_{2} \mathrm{eg}_{2}=$ bis(ethylene glycolato)diboron.

Acetonitrile was also proved to be a suitable solvent, although the yield of 4 was lower than that in THF solvent (entry 7).

Subsequently, the scope of this reaction was tested by employing various pyridine substrates. In general, this reaction exhibits a broad substrate scope with respect to the pyridine substrate, affording 1,4-DHP derivatives with different substitution patterns in moderate to good yields (Table 2). Although their structures were far from complex, many of these $N-\mathrm{H} \mathrm{1,4-}$ DHPs were synthesized for the first time, emphasizing the synthetic value of the present protocol. Generally, the yields of these rather air-labile products were quantified by ${ }^{1} \mathrm{H}$ NMR analyses of the reaction mixtures, and pure products could be obtained by flash column chromatography on basic alumina under an inert atmosphere. However, 4-substituted-1,4-DHPs were difficult to purify because they underwent rapid oxidation back to pyridine substrates $(\mathbf{4}, \mathbf{6 a}$, and $\mathbf{6 b})$. Electronwithdrawing substituents, such as amide, ester, and cyano groups, were proved beneficial to the hydroboration process, affording the 1,4-DHP products in good yields. ${ }^{10}$ Of special note, this inverse hydroboration protocol tolerated the active hydrogen in the pyridine substrates (e.g., 5b and 5c), which complements the traditional hydroboration methods. Multisubstituted pyridines, such as 2,5-, 3,4-, and 3,5-disubstituted ones (5h-1), were all compatible substrates. Interestingly, for a nicotinate substrate bearing a 4-methoxy substitution (5m), hydroboration occurred twice to afford 1,4-DHP product $6 \mathbf{6}$ when 2.2 equivalents of $\mathrm{B}_{2} \mathrm{eg}_{2}$ and base were employed, due to the elimination of the methoxy group. In all cases, the hydroboration occurred selectively at the 1,4-position, and no 1,2DHP product was obtained.

The successful umpolung of the pyridine hydroboration allowed for facile access to a series of 4-deuterated 1,4-DHPs. 1,4-DHPs are prevalently used as hydride and/or electron donors in organic chemistry, and 4-deuterium substituted 1,4DHPs are always employed as ideal mechanistic probes and organic deuteride transfer reagents. However, following the 
Table 2 Inverse hydroboration of pyridines ${ }^{a}$

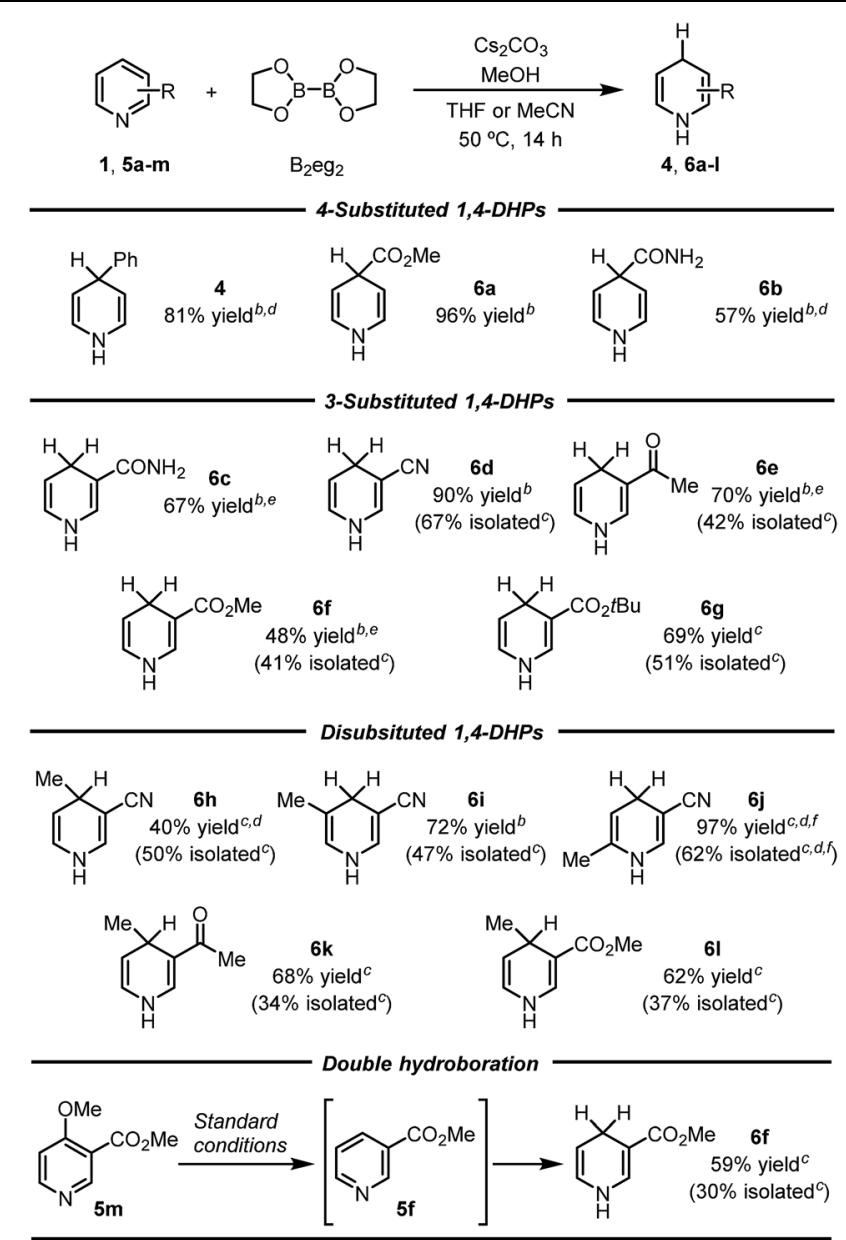

${ }^{a}$ Reaction conditions: pyridine substrate (1 equiv.), $\mathrm{B}_{2} \mathrm{eg}_{2}$ (1.1 equiv.), $\mathrm{Cs}_{2} \mathrm{CO}_{3}$ (1.1 equiv.), MeOH (11 equiv.), sealed tube, $50{ }^{\circ} \mathrm{C}$ for $14 \mathrm{~h}$. Yields of the 1,4-DHP products were determined by ${ }^{1} \mathrm{H}$ NMR analysis of the reaction mixture using DMSO as the internal standard (isolated yields are shown in parentheses). ${ }^{b}$ THF as the solvent. ${ }^{c}$ MeCN as the solvent. ${ }^{d} \mathrm{MeONa}$ was used instead of $\mathrm{Cs}_{2} \mathrm{CO}_{3} .{ }^{e} \mathrm{MeOK}$ and 18-crown6 (1.1 equiv. each) were used instead of $\mathrm{Cs}_{2} \mathrm{CO}_{3} .{ }^{f}$ The reaction was conducted at $65{ }^{\circ} \mathrm{C}$.

established synthetic methods (Scheme 1a-c), less accessible deuteride reagents (e.g., $\mathrm{NaBD}_{4}$ and DBpin) and deuterated aldehydes should be employed to prepare 4-deuterated 1,4DHPs, while the present inverse hydroboration protocol only requires a readily available $\mathrm{D}^{+}$source for the same task. The result shown in Fig. 1d has already demonstrated this, although the deutero ratio was still not satisfactory with only 2.2 equiv. of MeOD. To our delight, by employing 22 equiv. of MeOD, 1,4DHPs with high deuterium incorporation at the 4-position were obtained (Table 3).

It was found that most pyridine substrates described in Table 2 could be transformed to the desired 4-deuterated 1,4DHPs in moderate to good yields with excellent deuterium incorporation (up to $>98 \%$ ) at the C4 position. Furthermore, the structure of the 1,4-DHP product was unambiguously confirmed using the XRD structure of 3-cyano-4-deuterated-1,4-
Table 3 Synthesis of 4-deuterated 1,4-DHP derivatives ${ }^{a}$

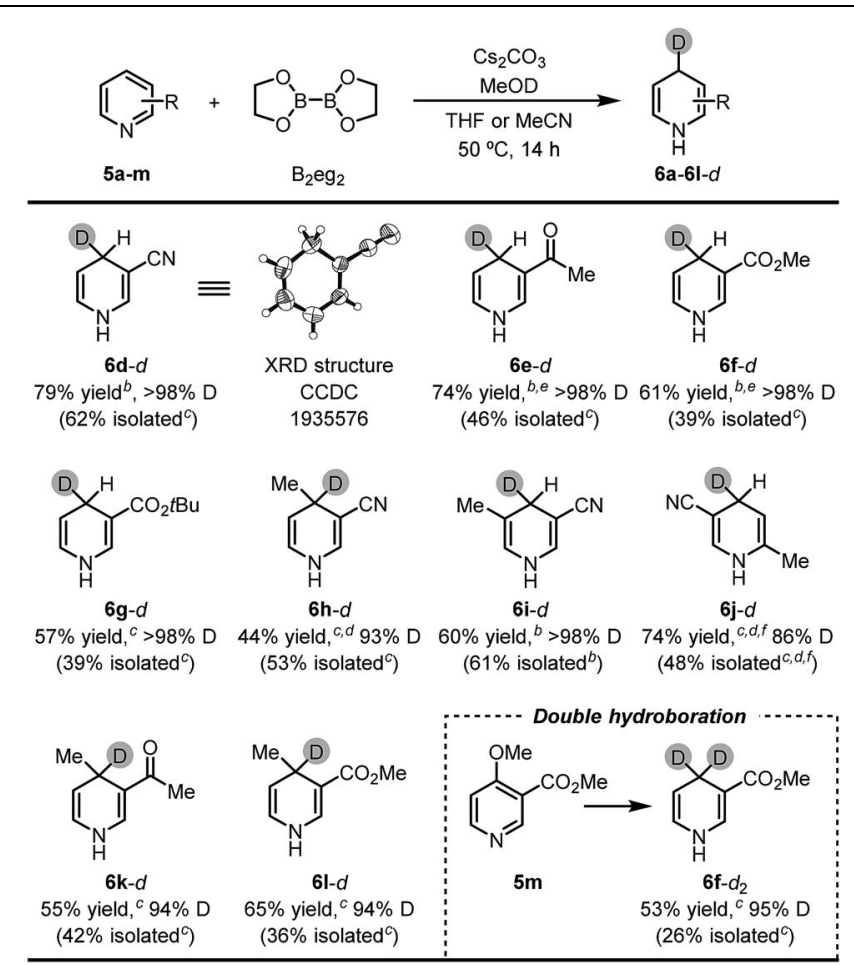

${ }^{a}$ Reaction conditions: pyridine substrate (1 equiv.), $\mathrm{B}_{2} \mathrm{eg}_{2}$ (1.1 equiv.), $\mathrm{Cs}_{2} \mathrm{CO}_{3}$ (1.1 equiv.), MeOD (22 equiv.), sealed tube, $50{ }^{\circ} \mathrm{C}$ for $14 \mathrm{~h}$. Yields of the 1,4-DHP products were determined by ${ }^{1} \mathrm{H}$ NMR analysis of the reaction mixture using DMSO as the internal standard (isolated yields are shown in parentheses). ${ }^{b}$ THF as the solvent. ${ }^{c} \mathrm{MeCN}$ as the solvent. ${ }^{d} \mathrm{MeONa}$ was used instead of $\mathrm{Cs}_{2} \mathrm{CO}_{3} .{ }^{e} \mathrm{MeOK}$ and 18-crown6 (1.1 equiv. both) were used instead of $\mathrm{Cs}_{2} \mathrm{CO}_{3} .{ }^{f}$ The reaction was conducted at $65^{\circ} \mathrm{C}$.

dihydropyridine $(\mathbf{6 d}-d)$. Interestingly, double inverse hydroboration of pyridine $\mathbf{5 m}$ has been successfully utilized to prepare 4,4-dideuterated 1,4-DHP $\mathbf{6 f}-d_{2}$. These results demonstrate that our method can provide a straightforward synthetic approach to a series of 4-deuterated 1,4-DHP derivatives, where easily available and inexpensive MeOD was used as the deuterium source.

Further experiments were also performed to reveal the limitations of the present protocol (Fig. 2). We found that halogenated pyridines $\mathbf{5 n}$ and 5o, 3-trifluoromethylpyridine (5p), 2-substituted pyridines $\mathbf{5 q}$ and $\mathbf{5 r}$, tetrasubstituted

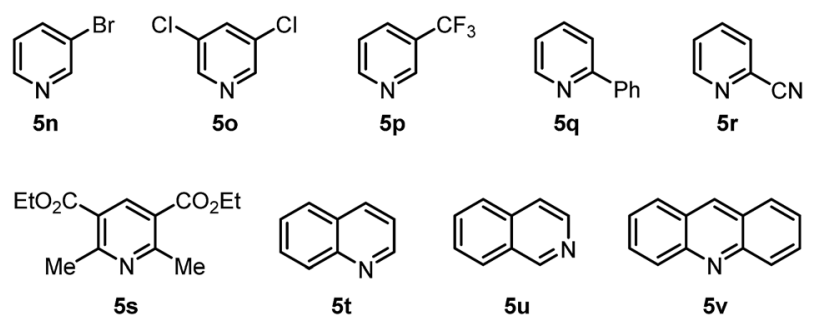

Fig. 2 Unsuccessful substrates for the inverse hydroboration. 


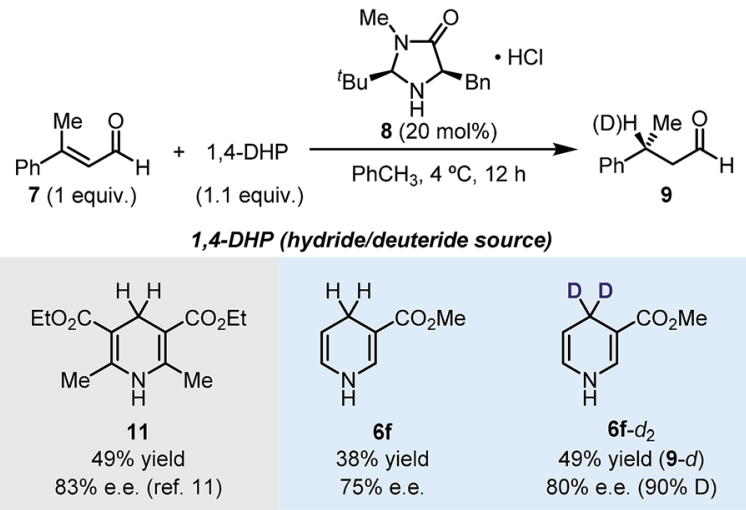

Scheme 4 The use of the produced 1,4-DHPs in asymmetric hydride/ deuteride transfer reaction.

pyridine $\mathbf{5 s}$, and other pyridine-embedded heterocycles, such as quinoline (5t), isoquinoline (5u) and acridine (5v), were not compatible with this inverse hydroboration protocol. They either exhibited a low reactivity, or produced a complex mixture of products under the reaction conditions.

\section{Potential use of the 1,4-DHP product}

1,4-DHPs are typical organic hydride donors in hydride transfer reactions. ${ }^{1 g}$ In particular, Hantzsch ester (HEH) is the most prevalently used hydride donor in asymmetric hydride transfer reactions. Since many of the newly synthesized 1,4-DHPs are structurally unprecedented in the literature, we are curious about their performance in asymmetric hydride transfer reactions as hydride donors. In this line, we explored the enantioselective hydride reduction of $\alpha, \beta$-unsaturated aldehyde 7 in the presence of organocatalyst 8 (Scheme 4). The model reaction employing HEH 11 as the hydride donor afforded chiral aldehyde 9 in $49 \%$ yield and $83 \%$ ee under literature conditions. ${ }^{11} \mathrm{~A}$ slight decrease in both yield and ee was observed when 1,4-DHP 6f was employed in place of HEH 11. Gratifyingly, when deuterium-labeled 1,4-DHP $\mathbf{6 f}-d_{2}$ was used, deuteride transfer occurred with comparable efficiency to hydride transfer $(50 \%$ yield, $80 \%$ ee), affording deuterium-enriched product $9(90 \%$ D). These results showcased the potential use of the 1,4-DHP products of the present method, which also indicated that in asymmetric hydride transfer reactions, the densely substituted DHP core of HEH is not a key factor in stereocontrol.

\section{Conclusions}

In conclusion, the umpolung of pyridine hydroboration has been realized for the first time by utilizing the diboron(4)/ pyridine/base system in a transition-metal-free manner. The key steps include a base-promoted formal addition of a boryl anion to pyridine, and the subsequent protonation of the produced $N$-boryl pyridyl anion complex. This inverse hydroboration process enables a simple and efficient method for the synthesis of 1,4-dihydropyridine derivatives from pyridines, and features broad substrate scope and good functional group compatibility. A great advantage of this protocol is that 4deuterated 1,4-DHPs could be easily prepared using easily available MeOD as the deuterium source. The produced 1,4DHP derivatives are complementary to previously known ones, and can act as useful organic hydride/deuteride donors.

\section{Conflicts of interest}

The authors declare no competing financial interest.

\section{Acknowledgements}

We acknowledge the financial support from the National Key Research and Development Plan (grant no. 2017YFA0505200) and the National Natural Science Foundation of China (grant no. 21772110 and 21822304). The technology platform of the CBMS and the Tsinghua Xuetang Talents Program are acknowledged for providing instrumentation and computational resources.

\section{Notes and references}

1 For selected reviews, see: (a) U. Eisner and J. Kuthan, Chem. Rev., 1972, 72, 1; (b) D. M. Stout and A. I. Meyers, Chem. Rev., 1982, 82, 223; (c) C. Safak and R. Simsek, Mini-Rev. Med. Chem., 2006, 6, 747; (d) N. Pollak, C. Dölle and M. Ziegler, Biochem. J., 2007, 402, 205; (e) N. Edraki, A. R. Mehdipour, M. Khoshneviszadeh and R. Miri, Drug Discovery Today, 2009, 14, 1058; (f) M. Rueping, J. Dufour and F. R. Schoepke, Green Chem., 2011, 13, 1084; $(g)$ C. Zheng and S. L. You, Chem. Soc. Rev., 2012, 41, 2498.

2 For selected reviews, see: (a) R. Lavilla, J. Chem. Soc., Perkin Trans. 1, 2002, 1141; (b) S. G. Ouellet, A. M. Walji and D. W. C. MacMillan, Acc. Chem. Res., 2007, 40, 1327; (c) A. Silva, E. Silva and P. Varandas, Synthesis, 2013, 45, 3053.

3 (a) E. E. Knaus and K. Redda, Can. J. Chem., 1977, 55, 1788; (b) D. L. Comins and A. H. Abdullah, J. Org. Chem., 1984, 49, 3392; (c) R. J. Sundberg, G. Hamilton and C. Trindle, J. Org. Chem., 1986, 51, 3672; (d) P. S. Wagenknecht, J. M. Penney and R. T. Hembre, Organometallics, 2003, 22, 1180; (e) J. A. Bull, J. J. Mousseau, G. Pelletier and A. B. Charette, Chem. Rev., 2012, 112, 2642; (f) Y. Maenaka, T. Suenobu and S. Fukuzumi, J. Am. Chem. Soc., 2012, 134, 367; (g) L. Q. Lu, Y. Li, K. Junge and M. Beller, Angew. Chem., Int. Ed., 2013, 52, 8382.

4 (a) J.-P. Wan and Y. Liu, RSC Adv., 2012, 2, 9763; (b) M. M. Khan, Saigal, S. Khan, S. Shareef and M. Danish, ChemistrySelect, 2018, 3, 6830.

5 For metal-catalysed hydroboration/hydrosilylation of pyridines, see: (a) L. Hao, J. F. Harrod, A.-M. Lebuis, Y. Mu, R. Shu, E. Samuel and H.-G. Woo, Angew. Chem., Int. Ed., 1998, 37, 3126; (b) R. Shu, J. F. Harrod, H.-G. Woo and E. Samuel, Can. J. Chem., 2001, 79, 1075; (c) D. V. Gutsulyak, A. van der Est and G. I. Nikonov, Angew. Chem., Int. Ed., 2011, 50, 1384; (d) M. Arrowsmith, M. S. Hill, T. Hadlington, G. Kociok-Kohn and C. Weetman, Organometallics, 2011, 30, 5556; (e) 
C. D. F. Königs, H. F. Klare and M. Oestreich, Angew. Chem., Int. Ed., 2013, 52, 10076; $(f)$ A. S. Dudnik, V. L. Weidner, A. Motta, M. Delferro and T. J. Marks, Nat. Chem., 2014, 6, 1100; $(g)$ J. Intemann, M. Lutz and S. Harder, Organometallics, 2014, 33, 5722; (h) J. Intemann, H. Bauer, J. Pahl, L. Maron and S. Harder, Chem.-Eur. J., 2015, 21, 11452; (i) J. Jeong, S. Park and S. Chang, Chem. Sci., 2016, 7, 5362; (j) A. Kaithal, B. Chatterjee and C. Gunanathan, Org. Lett., 2016, 18, 3402; ( $k$ ) J. L. Lortie, T. Dudding, B. M. Gabidullin and G. I. Nikonov, ACS Catal., 2017, 7, 8454; (l) S. Park and S. Chang, Angew. Chem., Int. Ed., 2017, 56, 7720; (m) F. Zhang, H. Song, X. Zhuang, C.-H. Tung and W. Wang, J. Am. Chem. Soc., 2017, 139, 17775; (n) P. Ji, X. Feng, S. S. Veroneau, Y. Song and W. Lin, J. Am. Chem. Soc., 2017, 139, 15600; (o) H. Liu, M. Khononov and M. S. Eisen, ACS Catal., 2018, 8, 3673; (p) S. R. Tamang, A. Singh, D. K. Unruh and M. Findlater, ACS Catal., 2018, 8, 6186; $(q)$ M. W. Gribble Jr, S. Guo and S. L. Buchwald, J. Am. Chem. Soc., 2018, 140, 5057; (r) J. Liu, J.-Y. Chen, M. Jia, B. Ming, J. Jia, R.-Z. Liao, C.-H. Tung and W. Wang, ACS Catal., 2019, 9, 3849.

6 For organocatalytic hydroboration/hydrosilylation of pyridines, see: (a) X. Fan, J. Zheng, Z. H. Li and H. Wang, J. Am. Chem. Soc., 2015, 137, 4916; (b) N. Gandhamsetty, S. Park and S. Chang, J. Am. Chem. Soc., 2015, 137, 15176; (c) Z. Y. Liu, Z. H. Wen and X. C. Wang, Angew. Chem., Int. Ed., 2017, 56, 5817; (d) E. N. Keyzer, S. S. Kang, S. Hanf and D. S. Wright, Chem. Commun., 2017, 53, 9434; (e)
J. E. Radcliffe, J. J. Dunsford, J. Cid, V. Fasano and M. J. Ingleson, Organometallics, 2017, 36, 4952; (f) B. Rao, C. C. Chong and R. Kinjo, J. Am. Chem. Soc., 2018, 140, 652; $(g)$ T. Hynes, E. N. Welsh, R. McDonald, M. J. Ferguson and A. W. H. Speed, Organometallics, 2018, 37, 841.

7 (a) L. Zhang and L. Jiao, J. Am. Chem. Soc., 2017, 139, 607; (b) L. Zhang and L. Jiao, Chem. Sci., 2018, 9, 2711; (c) L. Zhang and L. Jiao, J. Am. Chem. Soc., 2019, 141, 9124.

8 Bis-coordinated diboron(4) compounds have been characterized in previous studies, see: (a) S. Pietsch, U. Paul, I. A. Cade, M. J. Ingleson, U. Radius and T. B. Marder, Chem. - Eur. J., 2015, 21, 9018; (b) I. A. Cade, W. Y. Chau, I. Vitorica-Yrezabal and M. J. Ingleson, Dalton Trans., 2015, 44, 7506; For a review on diboron(4) compounds, see: (c) E. C. Neeve, S. J. Geier, I. A. I. Mkhalid, S. A. Westcott and T. B. Marder, Chem. Rev., 2016, 116, 9091.

9 A. Bonet, H. Gulyás and E. Fernández, Angew. Chem., Int. Ed., 2010, 49, 5130.

10 It was found that the pyridine substrate without an electronwithdrawing substituent could also undergo the hydroboration reaction, although affording a lower yield of the 1,4-DHP product. See the ESI $\dagger$ for details.

11 For selected examples, see: (a) J. W. Yang, M. T. Hechavarria Fonseca, N. Vignola and B. List, Angew. Chem., Int. Ed., 2004, 44, 108; (b) S. G. Ouellet, J. B. Tuttle and D. W. C. MacMillan, J. Am. Chem. Soc., 2005, 127, 32. 